\title{
Psychiatric Symptoms and Psychological Distress in Patients Suffering From Advanced Cancer and in Those Considered "Cured": The Role of Parenting and Family Relationship
}

\author{
Monica Pellerone \\ "Kore" University of Enna, Enna, Italy
}

\author{
Sandra Miccichè \\ Terzo Circolo "Chivasso", Chivasso (To), Italy
}

\begin{abstract}
The presence of psychiatric problems in cancer patients is related to the perception of social support, family involvement, and the disease stage. The advanced stage, dysfunctional social interactions, low parental support, and patients' youth can be considered as predictors of psychological comorbidity. The aim of this study is to analyze the parenting and perception of family support, as predictor variables to psychiatric symptoms and psychological distress in patients suffering from advanced cancer and in those considered "cured". The study, through cross-sectional design, involved an group of 30 patients with terminal cancer which are experts in the outpatient oncology, aged between 39 and 79 years (including 16 men and 14 women); a group of 27 people who have cured cancer, aged between 45 and 70 years (including 21 women and 6 men). They completed: MHQ (Middlesex Hospital Questionnaire), Parental Bonding Instrument, Demetra Test, and PDI (Patient Dignity Inventory). The results show that: Subjects with cured cancer have lower level of somatization than terminally ill patients; the former has a greater peace of mind, perception of social support and relational dependence. Our results seem to confirm the significant role of family involvement for the psychological distress.
\end{abstract}

Keywords: cancer, parenting, family, psychiatric symptoms, psychological distress

\section{Introduction}

The concept of healing in cancer patients is related to the time factor, since in most cases one cannot be certain that the disease has been entirely removed, and that there are no malignant cells in the body ready to give rise to local recurrences or metastases. For the different forms of cancer, it is generally considered cured the person who, 2-5-10 years after the end of treatment, does not show symptoms of disease anymore.

Patients with cancer have common psychiatric problems such as depression, anxiety and somatization, causing an alteration of the quality of life and the risk of failure in achieving life goals (Hack, Chochinov, Hassard, Kristjanson, McClement, \& Harlos, 2014; Pellerone \& Miccichè, 2014).

The presence of psychiatric problems in cancer patients, is related to the perception of social support received, the family involvement, and the disease stage (Vodermaier, Linden, MacKenzie, Greig, \& Marshall, 2011).

\footnotetext{
Monica Pellerone, Ph.D., Psychologist and Psychotherapist, Assistant Professor, Faculty of Humanities and Social Sciences, "Kore" University of Enna.

Sandra Miccichè, Psychologist, Teacher, Terzo Circolo "Chivasso", Chivasso (To).
} 
In detail, a positive relationship with mother seems to affect high levels of anxiety, an affective bond influences high levels of depression; a weak bond or anaffective brings high levels of somatization in cancer patient; also a constricting bond with father seems to affect high levels of anxiety and depression, a weak bond with the father brings high levels of somatic concomitants of anxiety and obsessional traits in the advanced cancer patient (Pellerone \& Miccichè, 2014).

Patients with advanced cancer need a better control of the disease, a great attention to their mental well-being and a psychological support from the members of their family. In detail, a recent research shows that social support and family have a positive effect on the quality of patient's life; otherwise anger, anxiety, worry, negative evaluation of the disease or low levels of social support have a negative effect (Julkunen, Gustavsson-Lilius, \& Hietanen, 2009; Northouse et al., 2002).

Literature, also, shows that the advanced disease stage, dysfunctional social interactions, low social support, the low level of education, and patient's youth can be considered as predictors of psychological comorbidity, above all anxiety, depression and post-traumatic stress disorder (Simon, Thompson, Flashman, \& Wardle, 2009).

\section{Psychiatric Symptoms and Social Support in Cancer Patients}

Common psychiatric problems such as depression, anxiety, demoralization and suicidal ideation, may arise during the lifetime of an cancer patient, from the communication of the diagnosis. Literature shows that social support is related to a greater psychological adjustment; family support is related to low levels of anxiety and depression at the hospital (Arora, Finney Rutten, Gustafson, Moser, \& Hawkins, 2007).

Furthermore, emotional support from family, friends and help on decision-making is associated with patients' health-related quality of life and self-efficacy outcomes (Neuling \& Winefield, 1988).

So, social support, as rated by patients, can be considered a significant predictor of changes in psychological distress (Moyer \& Salovey, 1999).

In addition, there are medical and psychosocial problems, including medication side effects, physical disability, addiction, bereavement, and dysfunctional family background, which helps to increase the risk of psychological and psychiatric disorders. Indeed, it was shown that psychiatric distress amplifies the perception of pain in subjects at the end-stage of cancer, and this leads to an increasing use of pharmacological interventions and lower quality of life.

The issue of depression with its treatment is one of the most studied areas in people with cancer, due the high prevalence and negative consequences. Many terminally ill patients describe a decrease in their ability to feel pleasure in their lives (Breitbart et al., 2002); in addition, the extreme psychological stress is a risk factor for suicidal ideation and suicide attempts, as well as requests for assisted suicide and the desire for hastened deat (Craparo, Gori, Mazzola, Petruccelli, Pellerone, \& Rotondo, 2014).

Depressed mood and sadness are emotional responses even for those patients on the mend, or in those who have successfully dealt with an intervention to eradicate the disease; although a recent research shows that the stage of disease is a predictor of depression, but only in men with lung cancer.

The incidence of clinical depression in people with cancer and its consequences have been extensively studied in years; recent investigations have shown that there are other dimensions of mental suffering related to depression, such as the syndrome of demoralization; it is a continuum between discouragement and deep despair, it's characterized by feelings of hopelessness, loss of meaning and purpose in life, feelings of lack of 
support, feeling trapped, personal bankruptcy dealing with a stressful situation, pessimism, lack of incentive or motivation to act differently (Clarke \& Kissane, 2001; Pellerone \& Miccichè, 2014).

Similarly to depression, doctors have found that high levels of anxiety goes with the terminal phase of an illness; anxiety can arise in response to stress factors, it can be a chronic condition, or it may be derived from sources such as iatrogenic, medical complications of the disease or treatments. Few studies have measured anxiety and attempted to identify and describe its pathological states, in patients with cancer. Because of the overlap between anxiety and depressive syndromes, which is well established in a population suffering from terminal illness, it's not always easy to distinguish these two disorders. The majority of depressed individuals also has considerable anxiety, although the reverse is not necessarily true.

Literature, moreover, shows that the level of anxiety is related to the variable of gender and disease stage: for example, Vodermaier and co-workers show that females with cancer exhibit more symptoms of anxiety and depression, compared to males; in addition, patients in advanced disease have higher levels of anxiety (Vodermaier, Linden, MacKenzie, Greig, \& Marshall, 2011).

Data research suggests that neurotic symptoms such as depression, and anxiety are as frequent if not more so than pain and other physical symptoms in palliative care settings. In such settings, the social and family support is fundamental (Jaiswal, Alici, \& Breitbat, 2014).

Unfortunately, however, while patients receive a high level of support during the closer period to the diagnosis and when approaching their death, the perception of the received support drops significantly within the first year of the disease. In fact, perceived helpfulness of information, the emotional and decision-making support provided by family, friends, and providers significantly decreases over time, above all within cured patients. The perception of reduced support enhances the distress psychological in the cured patient, such as anxiety and the sense of loneliness and helplessness, but this doesn't affect neurotic symptoms (Breitbart et al., 2002).

So, while recent literature has investigated the role of family support and friendship in people with cancer, few studies have shown these variables by comparing terminal cancer patients and cured people, or rather people who do not exhibit specific symptoms few years after the surgery. In fact, the neurotic symptoms and psychological distress during hospitalization can be alleviated by family, friendship and hospital support (such as palliative care); but the literature has neglected the study of neurotic and psychological symptoms in patients during the remission phase, in which such support drastically decreases.

The aim of this study is to analyze the parenting and the perception of family support, as predictor variables to psychiatric symptoms and psychological distress in patients suffering from advanced cancer and in those considered "cured".

According to the literature, we hypothesize:

(1) the presence of a significant relationship between family involvement and psychological distress in both groups of patients (Arora et al., 2007; Neuling \& Winefield, 1988);

(2) the presence of a relationship between parenting style and psychological distress only in the group of terminally ill (Pellerone \& Miccichè, 2014).

We have hypothesized that the absence of family support is predictive of neurotic symptoms only in the group of terminally ill, but it is predictive of psychological distress in both groups (Clarke \& Kissane, 2001). 


\section{Participants and Procedure}

The study population consists of: Group A of 30 patients with terminal cancer, aged between 39 and 79 years $(M=59.87 ; S D=14.90)$; this group includes 16 men with lung cancer and 14 with breast cancer.

Group B of 27 people who have cured cancer, aged between 45 and 70 years $(M=60.12 ; S D=9.45)$, including 21 women with breast cured cancer and 6 men with lung cured cancer.

The first group of patients with advanced terminal cancer (Group A) was recruited over a period of three months by a hospice in Sicily, that is a residential structure capable of housing terminally ill people, specializing in palliative care. These subjects came from Hospital Department of Oncology and were transferred to this structure because they don't have the opportunity to receive assistance in their homes; this group includes only hospitalized patients, with advanced terminal cancer.

The second group with cured cancer (Group B) was recruited over a period of six months by "Ados Vita" Association, in the city of Mazzarino and Riesi (Sicily-Italy). The "Ados Vita", association of Italian women breast surgery, includes women with breast cured cancer and men with forms of cured cancer. The study includes only patients with low risk of disease recurrence, who do not show specific symptoms of cancer after about three years since the surgery. Members of association were subjected to a program of follow-up (as established by the American Society of Clinical Oncology), which provides a checkup every three to six months for all subjects and hormone treatment with anti-estrogen for women who have had breast cancer.

All patients gave written consent before starting research and all study methods and materials were reviewed and approved by Hospital Department of Oncology and “Ados Vita” Association.

\section{Measures}

A cross-sectional research design has been used, choosing subjects with similar characteristics, such as women with breast cancer and men with lung cancer. Both groups were subjected to the administration of the same psycho-diagnostic instruments: MHQ, Parental Bonding Instrument, Demetra Test, and PDI.

All instruments were administered in person by a psychologist.

\section{MHQ (Crown \& Crisp, 1966)}

MHQ investigates the conventional diagnostic categories of psychoneurotic illness; it was originally used to diagnose symptoms due to excessive admissions in hospitals, but recently it has been used on the healthy population and not hospitalized to measure stress and neurotic behavior (Bagley, 2011). It is composed by 48 items divided into six steps which identify: FFA (Free-floating anxiety), PHO (Phobic anxiety), OBS (Obsessional traits and symptoms), SOM (Somatic concomitants of anxiety), DEP (Neurotic depression), and HYS (Hysterical personality traits);

\section{Parental Bonding Instrument (Parker, Tupling, \& Brown, 1979)}

Parental Bonding Instrument apt to evaluate the relationship that the patient has respectively with the mother and the father over the first 16 years of his/her life; it evaluates the relationship by following two axes: "care" (which could be characterized by an extreme of warmth or an extreme of coldness and indifference), and "overprotection" (which could be identified as an attitude of control and repression or as an attitude of self-worth);

\section{Demetra Test (Scrimali, 2005)}

Demetra Test tool apt to evaluate family relationship; it is composed by 20 items in order to estimate: the 
hostility, which leads to a lack of participation, and distress towards patient; the hyper-emotional, which leads the family to become overprotective and intrusive;

\section{PDI (Chochinov et al., 2008)}

PDI made up of 25 items that measure the dignity entity in five sub-scales: symptom distress, existential distress, dependence, sense of mental peace and support.

\section{Preliminary Analysis}

The administration of MHQ in the group of terminally ill patients shows that: Women themselves scored significantly higher than men in the size of hysteria $(F(1,29)=16.12 ; p<0.01)$.

Unlike in the group of subjects with cured cancer, gender differences emerge in the level of obsession $(F(1.26)=5.14 ; p<0.05)$ and somatization $(F(1,26)=4.82 ; p<0.05)$; the analysis of the mean scores showed that: Men show higher scores of women in the size of obsession and in that of somatization.

Tables 1 shows mean scores and standard deviations for all variables of MHQ in Group A and Group B.

Table 1

Mean Scores and Standard Deviations for All Variables of MHQ and Differences According to Sex

\begin{tabular}{|c|c|c|c|c|}
\hline \multirow{3}{*}{ Neurotic symptoms } & \multicolumn{2}{|c|}{ Group A } & \multicolumn{2}{|c|}{ Group B } \\
\hline & Men & Women & Men & Women \\
\hline & Mean $(S D)$ & Mean $(S D)$ & Mean $(S D)$ & Mean $(S D)$ \\
\hline FFA & $9.07(2.60)$ & $9.73(2.71)$ & $8.33(2.25)$ & $6.24(2.72)$ \\
\hline $\mathrm{OHO}$ & $7.87(1.55)$ & $6.80(1.90)$ & $7.87(1.55)$ & $7.76(2.59)$ \\
\hline OBS & $7.00(2.83)$ & $7.73(2.71)$ & $12.67(3.27)$ & $9.14(3.38)$ \\
\hline SOM & $11.60(2.75)$ & $11.13(1.41)$ & $12.20(3.77)$ & $8.00(3.84)$ \\
\hline DEP & $7.07(3.22)$ & $7.73(2.66)$ & $10.00(2.45)$ & $7.30(3.51)$ \\
\hline HYS & $1.40(1.45)$ & $3.67(1.63)$ & $4.00(1.41)$ & $4.30(2.74)$ \\
\hline
\end{tabular}

Notes. FFA = Free-floating anxiety; $\mathrm{PHO}=$ Phobic anxiety; OBS = Obsessional traits and symptoms; SOM = Somatic concomitants of anxiety; DEP $=$ Neurotic depression; HYS $=$ Hysterical personality traits.

The administration of PDI shows that in the group of terminally ill, women have significantly higher scores in the dimension of distress symptoms $(F(1,29)=4.37 ; p<0.05)$. In the group of subjects with cured cancer, instead, there is no difference due to gender variable.

Table 2 shows mean scores and standard deviations for all variables of PDI in the Group A.

Table 2

Mean Scores and Standard Deviations for All Variables of PDI and Differences According to Sex in Terminally Ill

\begin{tabular}{|c|c|c|c|c|}
\hline \multirow{2}{*}{ Dignity entity } & \multicolumn{2}{|c|}{ Men } & \multicolumn{2}{|c|}{ Women } \\
\hline & Mean & $S D$ & Mean & $S D$ \\
\hline Symptom distress & 11.40 & 4.21 & 14.67 & 4.35 \\
\hline Existential distress & 12.73 & 3.22 & 13.53 & 3.40 \\
\hline Mental peace & 7.20 & 1.90 & 7.27 & 2.34 \\
\hline Support & 7.20 & 2.34 & 8.13 & 1.25 \\
\hline Dependence & 13.60 & 2.23 & 13.47 & 2.88 \\
\hline Family hostility & 5.93 & 4.45 & 3.87 & 2.33 \\
\hline Family involvement & 16.40 & 4.91 & 14.93 & 1.36 \\
\hline
\end{tabular}


The administration of the Parental Bonding Instrument in Group B shows that gender significantly influences the level of care of the mother $(F=5.71, p<0.05)$ and father $(F=6.67, p<0.05)$ : Women perceive a higher level of maternal care (Men: $M=24.40, S D=45.41$; Women: $M=28.33, S D=3.38$ ) and paternal care (Men: $M=20.80, S D=8.16$; Women: $M=26.74, S D=3.56$ ).

The same instrument administered to terminally ill patients did not show differences due to the gender variable.

A $t$-test for independent groups was conducted in order to compare the scores obtained with the administration of the instruments in subjects cured (Group A) and in terminally ill patients (Group B). In detail:

(1) the assessment of neurotic symptoms shows that the two groups differ only in the levels of somatization $(F(1,53)=10.70 ; p<0.01)$ : The analysis of the average scores shows that Group A has lower scores than Group B (Group A: $M=8.84, S D=4.12$; Group B: $M=11.37 ; S D=2.16$ );

(2) the assessment of attachment shows that the two groups differ only in the perception of maternal $(F(1,51)=8.89 ; p<0.01)$ and paternal over-protection $(F(1,52)=9.22 ; p<0.01)$ : The subjects with cured cancer present average scores significantly higher in maternal control (Group A: $M=17.00, S D=7.00$; Group $\mathrm{B}: M=11.63, S D=3.69$ ) and paternal control (Group A: $M=18.29, S D=6.80$; Group B: $M=13.00, S D=$ 3.90);

(3) the evaluation of the levels of distress indicates that the two groups differ in (see Table 3): the level of existential distress $(F(1,55)=4.70 ; p<0.05)$, peace of mind $(F(1,55)=58.96 ; p<0.01)$, perception of support received $(F(1,55)=38.84 ; p<0.01)$ and relational dependence $(F(1,55)=31.64 ; p<0.01)$.

Table 3

Mean Scores and Standard Deviations of Distress in the Group A and Group B

\begin{tabular}{lcc}
\hline Dignity entity & Group A & Group B \\
\cline { 2 - 3 } & Mean $(S D)$ & Mean $(S D)$ \\
\hline Symptom distress & $14.44(5.24)$ & $13.03(4.52)$ \\
Existential distress & $15.56(4.52)$ & $13.13(3.28)$ \\
Mental peace & $14.22(5.91)$ & $7.23(2.10)$ \\
Support & $14.33(4.84)$ & $7.67(1.90)$ \\
Dependence & $16.38(5.78)$ & $13.53(2.53)$ \\
Family hostility & $6.74(3.37)$ & $4.90(3.64)$ \\
Family involvement & $13.63(4.32)$ & $15.67(5.05)$ \\
\hline
\end{tabular}

\section{Results}

The overall objective is to investigate the parenting style and the perception of family support as predictors to psychological distress and neurotic symptoms on terminal cancer patients and in those considered "cured".

The first objective is to evaluate the relationship between family involvement and psychological distress. In cured patients, the Pearson's correlation shows that the excessive family involvement is positively related to the perception of social support $(r=0.38 ; p<0.05)$.

Differently in the terminally ill: The hostility of the family negatively correlates with the peace of mind, social support and relational dependence; the family involvement is positively correlated with symptom distress, existential distress and perception of support (see Table 4).

A further objective is to verify the presence of the relationship between parenting style and psychological distress; the Pearson's correlation shows the absence of such relationship within the Group A; the same analysis 
conducted on terminally ill individuals noted that:

(1) the maternal care correlates positively with the level of existential distress $(r=0.45, p<0.05)$;

(2) paternal care-giving correlates with the symptom distress $(r=0.44 ; p<0.05)$ and with the perception of social support $(r=0.37, p<0.05)$.

The third objective was to identify predictive variables to neurotic symptoms; in the group of terminally ill, the multiple regression analysis shows that: Excessive hostility is predictive of levels of anxiety $(t=2.75, p<$ $0.05)$ with $56 \%$ of variance explained; it is predictive somatization too $(t=2.13, p<0.05)$ with $45 \%$ of variance explained; and it's a predictor of levels of depression $(t=2.14, p<0.05)$ with $49 \%$ of variance explained.

Within the group of cured subjects: The excessive hostility $(t=3.83, p<0.01)$ is a predictive variable of somatization $(64 \%$ explained variance); it is predictive $(t=4.20, p<0.01)$ of levels of obsession too $(67 \%$ variance).

Last goal is to identify predictors to psychological distress in both groups of participants; in the group of terminally ill patients, the multiple regression analysis finds that no variable for family support is predictive of psychological distress. In the group of cured subjects, instead, the regression analysis shows that:

(1) excessive involvement predicts family distress symptoms $(t=4.00, p<0.01)$ with $65 \%$ of variance explained, and the existential one $(t=2.60, p<0.01)$ with $48 \%$ of variance explained;

(2) excessive family hostility is a variable predictor to the low level of relational dependence $(t=-2.14, p$ $<0.01)$ with $51 \%$ of variance explained.

Table 4

Pearson's Correlation Coefficients in the Terminally Ill

\begin{tabular}{|c|c|c|c|c|c|c|c|}
\hline & a. & b. & c. & d. & e. & f. & g. \\
\hline a. Family hostility & - & & & & & & \\
\hline b. Family involvement & $0.384^{*}$ & - & & & & & \\
\hline c. Symptom distress & 0.170 & $0.641^{* *}$ & - & & & & \\
\hline d. Existential distress & 0.134 & $0.479^{*}$ & $0.732^{* *}$ & - & & & \\
\hline e. Mental peace & $0.446^{*}$ & 0.366 & $0.545^{* *}$ & $0.722^{* *}$ & - & & \\
\hline f. Support & $0.487^{* *}$ & $0.488^{* *}$ & $0.562^{* *}$ & $0.778^{* *}$ & $0.880^{* *}$ & - & \\
\hline g. Dependence & $0.477^{*}$ & 0.338 & $0.560^{* *}$ & $0.721^{* *}$ & $0.889^{* *}$ & $0.930^{* *}$ & - \\
\hline
\end{tabular}

Notes. ${ }^{* * *}$ Correlation is significant at the 0.01 level (2-tailed); ${ }^{*}$ Correlation is significant at the 0.05 level (2-tailed).

\section{Discussion}

The assessment of neurotic symptoms shows that subjects with cured cancer have lower level of somatization than terminally ill ones; in the same direction, the evaluation of psychological distress shows that subjects cured have a greater peace of mind, perception of social support and relational dependence.

The evaluation of parenting discloses that the two groups differ only in the perception of maternal and paternal over-protection; briefly, the group of cure subjects have parents with an strong attitude of control and repression.

Our study confirms the presence of a relationship between family involvement and psychological distress in both groups of patients, although there is a greater relation in the terminally ill one.

We believe that a traumatic event can cause a psychopathological reaction in the individual, although this reaction may be mediated by the role and involvement of the family and the style of parenting. In fact, 
according to the research hypothesis, our study shows a relationship between attachment style and parental psychological distress, even if only in the group of terminally ill.

Finally, confirming the hypothesis of the research, the study shows that the absence of family support is a predictive variable of neurotic symptoms in the group of terminally ill; and the absence of family support is predictive of psychological distress in both groups.

We agree with those who consider the importance of family support in the psychopathology of cancer patients.

\section{Conclusion}

Cancer is a multisystem disease that affects individual's body and the psyche, his history, his thoughts, feelings and relationships. Man's identity in fact arises in the womb, it develops within the first relational network that is family, our past and our future; only during adolescence identity becomes independent as well as the decision-making process and coping strategies to deal with the existential problems (Pellerone, 2013a; 2013b; 2014).

The family is the reality that precedes the disease. It's the first group that recognizes the suffering of the patient integrating the different skills of each member to address the cancer event. Interventions addressing depression, hopelessness, and family support appear to be important aspects of adequate palliative treatments, particularly as it relates to the desire of a hastened death.

Our results show that a cured patient, who benefits from the familiar and social support, faces the disease in a more positive way, with good effects on his/her quality of life and psychological wellness.

Different is the situation of terminal ill patients, which combines a excessive family support with a high level of parental care, because this will have a negative effect on the disease and the existential distress; even though the absence of family support is a predictive variable of neurotic symptoms. In fact, terminal patients can manifest the freezing emotional, a defensive barrier built between the patient himself and the external environment; such create an emotional distance from the family in order to protect them and other relatives from the pain of a possible loss. This kind of reaction becomes stronger for late stages of disease.

The authors declare that they have full control of the primary data and agree to allow the journal to review these data. The authors report no other conflicts of interest in this work.

\section{References}

Arora, N. K., Finney Rutten, L. J., Gustafson, D. H., Moser, R., \& Hawkins, R. P. (2007). Perceived helpfulness and impact of social support provided by family, friends, and health care providers to women newly diagnosed with breast cancer. Psychooncology, 16(5), 474-86.

Bagley, C. (2011). The factorial reliability of the Middlesex Hospital Questionnaire in normal subjects. The British Psychological Society, 53(1), 53-58.

Breitbart, W., Rosenfeld, B., Pessin, H., Kaim, M., Funesti-Esch, J., Galietta, M., .. Brescia, R. (2002). Depression. hopelessness. and desire for hastened death in terminally ill patients with cancer. Journal of the American Medical Association, 284(22), 2907-2911.

Chochinov, H. M., Hassard, T., McClement, S., Hack, T., Kristianson, L., Harlos, M., .. Murray, A. (2008). The Patient Dignity Inventory: A novel way of measuring dignity-related distress in palliative care. Journal of Pain and Symptom Management, $36(6), 559-571$.

Clarke, J. D. M., \& Kissane, D. W. (2001). Demoralization: Its phenomenology and importance. Australian and New Zealand Journal of Psychiatry, 36(6), 733-742. 
Craparo, G., Gori, A., Mazzola, E., Petruccelli, I., Pellerone, M., \& Rotondo, G. (2014). Posttraumatic stress symptoms: Dissociation and alexithymia in an italian sample of flood victims. Neuropsychiatric Disease and Treatment, 10, $2281-2284$.

Crown, S., \& Crisp, A. H. (1966). A short clinical diagnostic self rating-scale for psychoneurotic patients: The Middlesex Hospital Questionnaire (MHQ). Brit. J. Psychiat., 112, 917-923.

Hack, T. F., Chochinov, H. M., Hassard, T., Kristjanson, L. J., McClement, S., \& Harlos, M. (2014). Defining dignity in terminally ill cancer patients: A factor-analytic approach. Psychooncology, 13, 700-708.

Jaiswal, R., Alici, Y., \& Breitbat, W. (2014). A comprehensive review of palliative care in patients with cancer. International Review of Psychiatry, 26(1), 87-101.

Julkunen, J., Gustavsson-Lilius, M., \& Hietanen, P. (2009). Anger expression: Partner support and quality of life in cancer patients. Journal of Psychosomatic Research, 66(3), 235-244.

Moyer, A., \& Salovey, P. (1999). Predictors of social support and psychological distress in women with breast cancer. Journal of Health Psychology, 4, 177-191.

Neuling, S. J., Winefield, H. R. (1988). Social support and recovery after surgery for breast cancer: Frequency and correlatees of supportive behaviours by family, friends and surgeon. Soc Sci Med., 27(4), 385-392.

Northouse, L. L., Mood, D., Kershaw, T., Schafenacker, A., Mellon, S., Walker, J., ...Decker, V. (2002). Quality of life of women with recurrent breast cancer and their family members. Journal of Clinical Oncology, 20(19), 4050-4064.

Parker, G., Tupling, H., \& Brown, L. B. (1979). Parental Bonding Instrument (PBI). British Journal of Medical Psychology, 52, 1-10.

Pellerone, M. (2013a). Identity status: Coping strategy and decision making process among Italian university students. Journal of Procedia-Social and Behavioral Sciences, 106, 1399-1408.

Pellerone, M. (2013b). Time perception in children with developmental dyscalculia. Journal of Procedia-Social and Behavioral Sciences, 103, 1220-1227.

Pellerone, M. (2014). Interpersonal adjustment, personal resources, aptitudes and interests: Vocational decision making process in a group of Italian adolescents. US-China Education Review, 4(9b), 648-655.

Pellerone, M., \& Miccichè, S. (2014). Prenatal attachment and anxiety: Women who decide to try in vitro fertilization and women who procreate naturally. Psychology Research, 4(6), 419-427.

Pellerone, M., \& Schimmenti, V. (2011). Status of identity interests and decision-making styles: Adolescents and the process of vocational choice. Giornale Italiano Di Psicologia Dell'Orientamento, 12, 27-37.

Scrimali, T. (2005). Demetra test: An instrument to measure the emotional context of family. Enna: Istituto Superiore per le Scienze Cognitive Editore.

Simon, A. E., Thompson, M. R., Flashman, K., \& Wardle, J. (209). Disease stage and psychosocial outcomes in colorectal cancer. Colorectal Disease, 11(1), 19-25.

Vodermaier, A., Linden, W., MacKenzie, E., Greig, D., \& Marshall, C. (2011). Disease stage predicts post-diagnosis anxiety and depression only in some types of cancer. British Journal of Cance., 105, 1814-1817. 